\title{
Total 3s Emission Yield as Bulk-Sensitive Probe for a True Soft X-ray Absorption Spectrum?
}

\author{
Piter S. Miedema*(i) and Martin Beye ${ }^{(i)}$ \\ DESY Photon Science, FS-FLASH, Notkestraße 85, 22607 Hamburg, Germany
}

\section{Supporting Information}

ABSTRACT: The detection of the true soft X-ray absorption typically needs specially prepared submicrometer thin samples for transmission measurements. Bulk experiments instead have to rely on yield methods, for example, electron yield with limitations for insulating samples, sensitivity to applied fields, and with limited bulk sensitivity. Fluorescence yield methods instead do not have those limitations but have been found to deviate, in general, from the absorption spectrum. We demonstrate that restricting the detection to the $3 \mathrm{~s}$ fluorescence channel (with the detector at a special angle where all polarizations contribute equally) restores the true X-ray absorption spectrum for all $3 \mathrm{~d}$ metal $L_{2,3}$ edges. These theoretically derived results are rationalized by the lack of $3 s-3 d$ interaction in the core-excited state. Comparing X-ray absorption versus 3s-PFY for arbitrary detection geometries for both linear and circular polarized light, deviations appear that can become as large as $15 \%$.

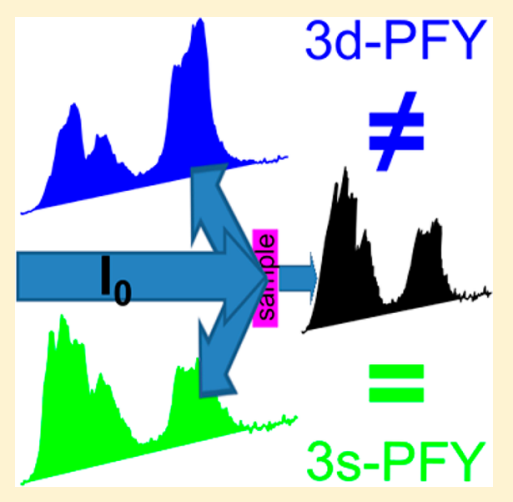

$\mathrm{X}$ -ray absorption spectroscopy has become a standard tool for electronic structure analysis of materials, ${ }^{1}$ whereas the small line widths in the soft $\mathrm{X}$-ray range combine element specificity with the best potential energy resolution for all elements. ${ }^{2}$ The $3 \mathrm{~d}$ transition-metal $\mathrm{L}_{2,3}$ edges (where a $2 \mathrm{p}$ electron is mainly excited into unoccupied $3 d$ states: $2 \mathrm{p}^{6} 3 \mathrm{~d}^{n} \rightarrow$ $2 \mathrm{p}^{5} 3 \mathrm{~d}^{n+1}$ ) provide direct information on the $3 \mathrm{~d}$ electronic structure in those materials, relevant, for example, for catalysis, ${ }^{3}$ magnetism, and magnetic storage $\mathrm{e}^{4}$ as well as battery applications. ${ }^{5}$ Soft X-rays tuned to energies at specific absorption edges do not deeply penetrate into matter, restricting transmission measurements of the sample absorption to specially prepared submicrometer thin specimens in vacuum. For studies on thick samples, indirect yield methods provide an alternative. The drain current from the sample after X-ray excitation is often used: The soft X-ray excitation leads to the emission of electrons. The now charged sample neutralizes by attracting electrons. This current is measured and is largely proportional to the original soft X-ray excitation, that is, the true X-ray absorption. Because of the limited electron escape depth from the sample, drain current measurements are a surface-sensitive probe of X-ray absorption with limitations for insulating samples and problems in electric and magnetic fields. Detecting the fluorescence yield instead provides a more bulklike probe of X-ray absorption.

With these alternative indirect methods, one assumes that there is no variation in the respective yield of emission over the studied energy range. However, it is known that this assumption is incorrect for fluorescence yield on systems with strong local correlations. ${ }^{6}$ Optical effects like saturation due to similar penetration and escape depths of the X-ray photons can influence fluorescence yield spectra and depend particularly on the experimental geometry. In addition, the measurement of the total fluorescence yield (TFY) can be affected by strong resonances at energies lower than the investigated absorption edge. In liquid solutions, ${ }^{7-10}$ but also in solid $\mathrm{Fe}_{2} \mathrm{O}_{3}{ }^{7}$ measurements of the TFY-X-ray absorption spectrum (XAS) display intensity dips. They are explained with the dominant oxygen $2 p$ emission being decreased by the onset of absorption at the transition metal. This decrease is only partially compensated by emission from the metal. It was emphasized before that measuring the fluorescence yield XAS via the total $3 \mathrm{~d}$ to $2 \mathrm{p}$ emission, referred to as the $3 \mathrm{~d}$ partial fluorescence yield (PFY), provides all X-ray absorption features but that there are intensity differences that directly relate to the on-site electronic correlations. ${ }^{8,11}$ In particular, the intensity of the $\mathrm{L}_{3}$ edge in 3d-PFY-XAS is lower than expected. ${ }^{11}$ This hinders for example using the branching ratio between the $L_{2}$ and $L_{3}$ edges measured with 3d PFY-XAS to gain information about spin states. ${ }^{12}$ On a side note, we want to stress that we consider here the sum of all $3 \mathrm{~d}$ to $2 \mathrm{p}$ ( $3 \mathrm{~s}$ to $2 \mathrm{p}$ ) emission channels for the $3 \mathrm{~d}$ (3s-) partial fluorescence yield (PFY), in contrast with the "High Energy Resolution Fluorescence Detection" (HERFD) method, ${ }^{1}$ where only parts of one channel are considered and even larger (in some cases desirable) deviations from XAS are expected. More recently, Golnak et al. $^{13}$ as a followup to Soldatov et al., ${ }^{10}$ have shown that 3 s-PFY for the $\mathrm{FeCl}_{3}$ and $\mathrm{CoCl}_{2}$ aqueous solutions, respectively (effectively $\mathrm{Fe}^{3+}\left(\mathrm{H}_{2} \mathrm{O}\right)_{6}$ and $\left.\mathrm{Co}^{2+}\left(\mathrm{H}_{2} \mathrm{O}\right)_{6}\right)$, appears to provide an "undistorted" or true XAS or at least provides the correct $\mathrm{L}_{3} / \mathrm{L}_{2}$ branching ratio.

In this Letter, we show theoretically that 3s-PFY can indeed restore the true XAS, albeit only when a special experimental

Received: March 8, 2018

Accepted: May 1, 2018

Published: May 1, 2018 


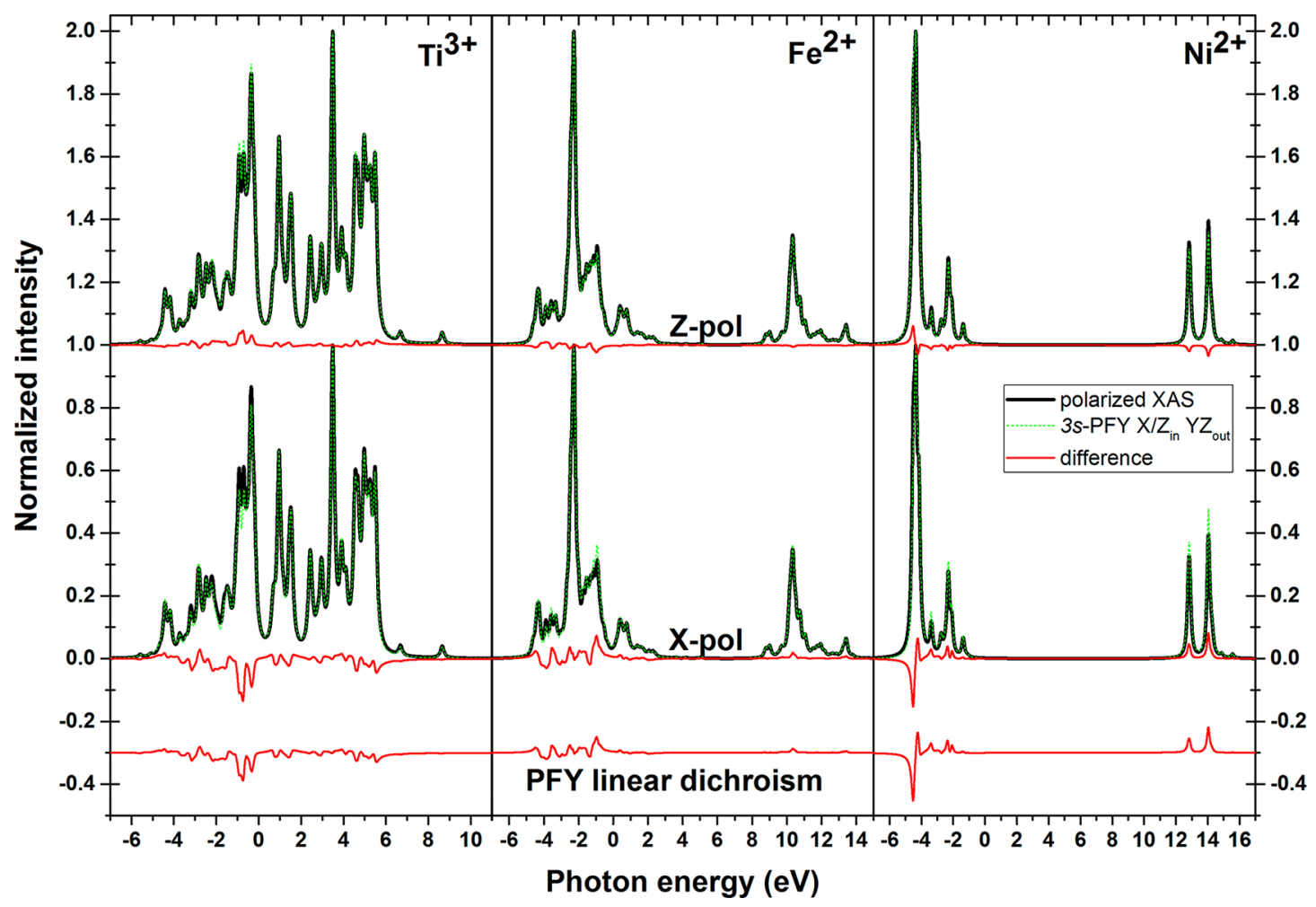

Figure 1. Vertically (Z-, top, offset by 1$)$ and horizontally (X-, middle) polarized absorption spectra of true XAS (black) and 3s-PFY including only the 3 s fluorescence of the detectable $Y$ - and $Z$-polarized components (green dotted) for $\mathrm{Ti}^{3+}$ (left), $\mathrm{Fe}^{2+}$ (middle), and Ni ${ }^{2+}$ (right) in $\mathrm{O}_{h}$ symmetry with $10 \mathrm{Dq}=0.6^{*}$ (oxidation state). All spectra are normalized to the highest peak. The red lines in the top and middle indicate the difference between 3s-PFY and XAS for the different $Z$ and $X$ polarizations. At the bottom (offset by -0.3 ), the remaining 3 s-PFY linear dichroism of $X-Z$ polarized component (absent in the true XAS) is shown for $\mathrm{Ti}^{3+}$ (left), $\mathrm{Fe}^{2+}$ (middle), and $\mathrm{Ni}^{2+}$ (right) in $\mathrm{O}_{h}$ symmetry with $10 \mathrm{Dq}=0.6^{*}($ oxidation state).

geometry is used. First, we rationalize the absence of intensity differences with the absence of the influence of on-site electronic correlations in the XAS excited state due to the closed $3 \mathrm{~s}$ shell in the case in which isotropic X-ray absorption and $3 \mathrm{~s}$ emission of $3 \mathrm{~d}$-metal ions in an octahedral $\left(O_{h}\right)$ crystal field is considered. Thereafter, simulations of more experimentally relevant situations, such as in the experiments of Golnak $^{13}$ and Soldatov et al., ${ }^{10}$ are discussed in which the detection geometry suppresses certain fluorescence polarizations. We show that differences between true XAS and 3sPFY may then actually occur.

First, we will start with discussing calculations in the isotropic approximation; for example, all polarizations of the incoming and outgoing photons are equally added. We have done calculations for the $3 \mathrm{~d}$ fillings $3 \mathrm{~d}^{1}$ to $3 \mathrm{~d}^{8}$. We have considered the $3 \mathrm{~d}^{9}$ system (for example $\mathrm{Cu}^{2+}$ ) to be unnecessary because the filled $d$ shell in the X-ray absorption final state $\left(2 \mathrm{p}^{5} 3 \mathrm{~d}^{10}\right)$ does not have $3 d-3 d$ correlations and therefore no differences between XAS and any PFY-XAS are expected.

All simulated spectra with isotropic X-ray absorption and $3 \mathrm{~s}$ fluorescence revealed that 3s-PFY-XAS and XAS are completely identical after normalization. (See the Supporting Information.) This result is substantiated as follows: $3 \mathrm{~d}-\mathrm{PFY}$ deviates from true XAS because of an additional $2 p-3 d$ exchange correlation effect entering in the $3 \mathrm{~d} \rightarrow 2 \mathrm{p}$ core-hole fluorescence decay. ${ }^{11}$ For 3s-PFY instead, the exchange correlations do not appear in the $3 \mathrm{~s} \rightarrow 2 \mathrm{p}$ fluorescence decay step because the completely filled $3 \mathrm{~s}$ shell in the core-excited state is not influenced by those correlations. Nevertheless, after $3 \mathrm{~s}$ to $2 \mathrm{p}$ fluorescent decay, correlations between $3 \mathrm{~s}$ and $3 \mathrm{~d}$ occur but only result in a redistribution of spectral weight inside the $3 \mathrm{~s}$ decay channel while not influencing the total $3 \mathrm{~s}$ emission, as measured in $3 \mathrm{~s}$ PFY. This argument is valid as long as the $3 \mathrm{~s}$ subvalence states are completely filled in the ground and core excited states, which is the case when they are at sufficiently lower energy than the $3 \mathrm{~d}$ valence states. For the main group elements in the third period, the $3 \mathrm{~s}$ level contributes to the valence states and will thus show deviations between 3s-PFY and XAS. For the $3 \mathrm{~d}$ transition metals and even heavier elements, the $3 \mathrm{~s}$ levels are separated by $>20 \mathrm{eV}$ from the Fermi level, and no differences between 3s-PFY and true XAS are expected.

Because for all of the $3 \mathrm{~d}$-metal $\mathrm{L}_{2,3}$ absorption edges $3 \mathrm{~s}$ emission is present, $3 \mathrm{~s}-\mathrm{PFY}$ can become a more universal yield measure of $3 \mathrm{~d}$-metal materials for obtaining the true XAS, ${ }^{13}$ also when compared with the commonly used inverted oxygen $2 p$ emission, ${ }^{5,9,10}$ because the latter is only possible if oxygen is present in the sample and when the 3d-metal edge of interest is above the oxygen K-edge. We note, though, that compared with $3 \mathrm{~d}$-PFY, $3 \mathrm{~s}$ emission is weaker than $3 \mathrm{~d}$ emission because of the smaller number of electrons and smaller transition matrix elements.

In experiments, the outgoing fluorescence may get partially absorbed inside the sample. This effect is termed selfabsorption (also sometimes mentioned as part of saturation effects) and is especially strong when the $3 \mathrm{~d}$ emission is energetically close to the resonant $\mathrm{L}_{2,3}$-edge absorption edge. The strong variations in the absorption of the fluorescence while penetrating through the sample to the detector effectively distort the measured yield (but can be corrected for with known spectral distributions ${ }^{9}$ ). For $3 s$-PFY this effect can be 
largely neglected because the emission energy is sufficiently separated in energy from the actual absorption resonance.

In perfect $O_{h}$ symmetry, there is no difference between the different spatial dimensions, and thus for the X-ray absorption, isotropic XAS is the same as the horizontally $(X-)$ polarized XAS and vertically ( $Z$-) polarized XAS. For our simulations, we have chosen the $Y$ direction as the incoming beam direction. Calculating 3s-PFY with one of these incoming polarizations while using isotropic 3s fluorescence (adding the $X$-, $Y$-, and $Z$ polarized fluorescence), the 3s-PFY spectrum still matches the true XAS (Figure S2 in the Supporting Information) for the different polarizations.

In our theoretical treatment we can extract the different geometry components $X, Y$, and $Z$ independently for both the incoming (absorbed) and outgoing (fluorescent) X-rays. In $O_{h}$ symmetry, there are only two inequivalent combinations of incoming and outgoing polarizations: the polarization components, where the incoming and outgoing polarization are parallel $\left(X_{\text {in }} X_{\text {out }}=Y_{\text {in }} Y_{\text {out }}=Z_{\text {in }} Z_{\text {out }}=A\right)$, and the crossed polarization components $\left(X_{\text {in }} Y_{\text {out }}=X_{\text {in }} Z_{\text {out }}=Y_{\text {in }} X_{\text {out }}=Y_{\text {in }} Z_{\text {out }}=\right.$ $\left.Z_{\text {in }} X_{\text {out }}=Z_{\text {in }} Y_{\text {out }}=B\right)$ for $O_{h}$ symmetry.

Now, let us consider a typical experimental geometry, where the detector (e.g., a RIXS spectrometer) is horizontally positioned at $90^{\circ}$ to the incoming beam (which impinges along $Y$ ) with polarization along $X$ (horizontal polarization), like for the experiments of Golnak ${ }^{13}$ and Soldatov et al. ${ }^{10}$ The $X$-polarized fluorescence component originates from a dipole oscillating parallel to the $X$ axis. Such a dipole has an emission node in the $X$ direction and thus will not appear on the detector positioned along the $X$ direction. For this particular case, the simulation results of XAS and 3s-PFY for $\mathrm{Ti}^{3+}, \mathrm{Fe}^{2+}$, and $\mathrm{Ni}^{2+}$ in $O_{h}$ symmetry are shown in Figure 1 . We note here that for these simulations the rule of thumb of $10 \mathrm{Dq}=0.6^{*}$ oxidation state $^{14}$ was used, which agrees often very well with experimental data with particular formal oxidation states, especially for oxides. $^{1,15}$

At a first glance, these symmetry-selected simulated 3s-PFY spectra (green dotted lines) largely overlap with the simulated XAS spectra (black lines) for $\mathrm{Ti}^{3+}, \mathrm{Fe}^{2+}$, and $\mathrm{Ni}^{2+}$ in $\mathrm{O}_{h}$ symmetry shown in Figure 1. Again, this proves that 3s-PFY with a detector at any angle might indeed be useful in branching ratio determination for spin-state analysis. ${ }^{12}$ However, there are deviations in both the horizontally $(X-)$ polarized (up to $15 \%$ ) and vertically (Z-) polarized 3s-PFY absorption spectra compared with the true XAS, as can be seen in the difference plots (red lines in top and middle of Figure 1). In addition, the differences for both incoming polarizations are not compensated and are larger when only crossed polarizations contribute $\left(X\right.$-polarized in, measured $X_{\text {in }} Y_{\text {out }}+X_{\text {in }} Z_{\text {out }}=$ $2 B$, red lines, middle), as opposed to the $Z$-polarized spectra $\left(Z_{\text {in }} Y_{\text {out }}+Z_{\text {in }} Z_{\text {out }}=A+B \neq 2 B\right.$, red lines, top), where crossed and parallel polarizations are detected (although with different weights than in the isotropic case, where it would be $A+2 B$ in $\mathrm{O}_{h}$ symmetry). This leads to a substantial linear dichroism (Figure 1, red lines bottom; $\mathrm{Ni}^{2+}$, for example, shows a dichroism up to 20\%) that is absent for true XAS in $O_{h}$ symmetry. These simulations also show that for the experiments of Soldatov ${ }^{10}$ and Golnak et al., ${ }^{13}$ their horizontally polarized 3s-PFY might have still deviated from the true XAS by up to $15 \%$ in particular peaks, while maintaining the correct branching ratio within the experimental noise. Thus 3s-PFY leads to an apparent linear dichroism in $O_{h}$ symmetry when the detector is at $90^{\circ}$ with respect to the incoming beam (in the direction of the horizontal polarization $X$ ).

To recreate a situation where the detector angle allows for a correct measurement of the true XAS in $O_{h}$ symmetry, the detector has to be specially placed: The angle has to be chosen such that the differently polarized outgoing 3s-fluorescence channels (the $X-, Y$-, and $Z$-polarized components) are detected with equal contribution. In general, with $\alpha$ and $\theta$ being the spherical angles ( $\alpha$, the angle within the $X Y$ plane; $\theta$, the angle to the $Z$ axis), the detected fluorescence intensity is $I=$ $X_{\text {out }}{ }^{*} \sin ^{2}(\alpha) * \sin ^{2}(\theta)+Y_{\text {out }} * \cos ^{2}(\alpha) * \sin ^{2}(\theta)+Z_{\text {out }} * \cos ^{2}(\theta)$. To guarantee that all components are registered with equal intensity, the angular prefactors have to be the same. This is fulfilled for $\sin (\alpha)=\cos (\alpha)$, that is, $\alpha=45^{\circ}$ and $\sin ^{2}(\theta)=2 / 3$, that is, $\theta$ at the magic angle to the $Z$ axis $\sim 54,7^{\circ}$. When the detector is located at this specific position, one can recover the $3 \mathrm{~s}-\mathrm{PFY}$ in a "pseudo-isotropic fluorescence situation", and, as a consequence, $3 \mathrm{~s}$-PFY spectra are representing the true XAS for linear dichroism studies. We point out that this solution not only holds for $O_{h}$ symmetry but also may be considered valid for any symmetry.

It is also interesting to analyze how the X-ray magnetic circular dichroism (XMCD) signal behaves for 3s-PFY versus true XAS because a recent paper has shown that there are problems with the sum rules from XMCD with 3d-PFY/TFY detection. ${ }^{16} \mathrm{XMCD}$ simulations of true XAS and $3 s$-PFY for $\mathrm{Ti}^{3+}, \mathrm{Fe}^{2+}$, and $\mathrm{Ni}^{2+}$ in $\mathrm{O}_{h}$ symmetry with a magnetic field of $B=$ $10 \mathrm{~T}$ are shown in Figure 2. The XMCD signal and shape for 3sPFY with isotropic or "pseudo-isotropic" 3s fluorescence conditions (green dotted lines, Figure 2) are observed to be the same as the true XAS (black lines).

For 3s-PFY with only the 3 s fluorescence from the $Y$ - and $Z$ polarization components (with a fluorescence yield detector at $90^{\circ}$ to the incoming beam; red lines in Figure 2), the signal not only is enhanced but also seems to contain features that are not present in the standard XMCD (black lines). In particular, for $\mathrm{Ni}^{2+}$, this is very apparent because the red line even moves out of the window and there is a nonzero XMCD at $-7 \mathrm{eV}$, below the $\mathrm{Ni}_{2,3}$ edge. Overall, the red lines seem to have an inverse linear dichroism component (see red lines in top of Figure 1) adding up to the XMCD signal.

Summarized with simple arguments and dedicated calculations and backed up with experimental data from refs 10 and 13, we rationalize how 3 s-PFY can be used as a sensitive yield method to measure the true bulk-sensitive XAS at the $3 \mathrm{~d}$-metal $\mathrm{L}_{2,3}$ edges in the isotropic fluorescence approximation. However, in real-life experiments, the X-ray polarization and, for example, the orientation of the detector with respect to the incoming beam are important as well. We have shown that $3 \mathrm{~s}$ PFY may then lead to substantial differences as compared with the true XAS. We discussed that these differences can be overcome by selecting the right angle for the detector to create a "pseudo-isotropic fluorescence situation". In addition, the 3sPFY probe gives the correct branching ratio (e.g., same as true $\mathrm{XAS}$ ) in contrast with $3 \mathrm{~d}-\mathrm{PFY}$. $^{11}$

With recent developments in PFY detection with transitionedge sensors ${ }^{17}$ or with efficient fluorescence energy separation via transmission or reflection zone plates, ${ }^{18,19} 3 \mathrm{~s}$ - and $3 \mathrm{~d}-\mathrm{PFY}$ separation will be rather straightforward in experiments. We anticipate that 3s-PFY-XAS could become a standard yield method to measure dilute active centers in an inactive matrix with applications in catalysis, (photo-) chemistry, and biology. For linear dichroism and circular dichroism studies with $3 \mathrm{~s}$ - 


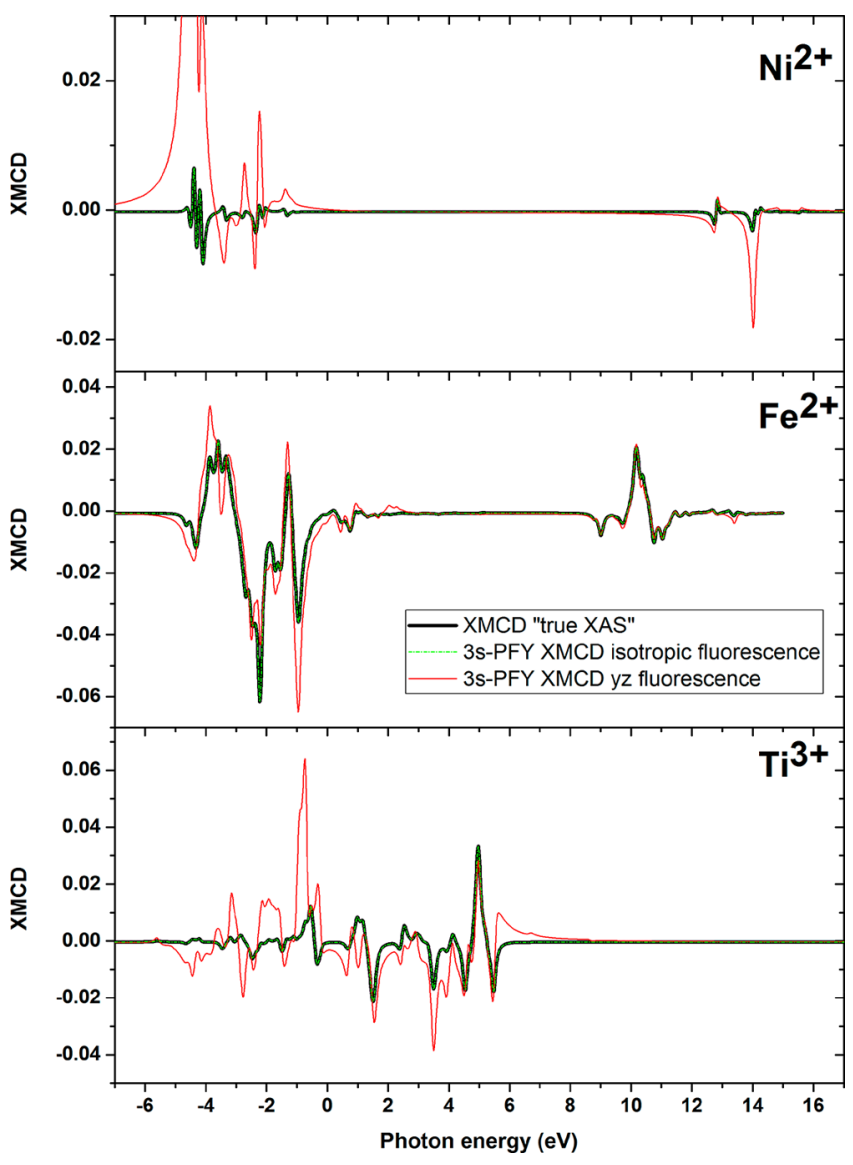

Figure 2. XMCD simulations of true XAS (black), 3s-PFY using isotropic fluorescence (green), and 3s-PFY using only 3s-fluorescence from the $\mathrm{Y}$ - and Z-polarized components (red) for $\mathrm{Ti}^{3+}, \mathrm{Fe}^{2+}$, and $\mathrm{Ni}^{2+}$ in $O_{h}$ symmetry with $10 \mathrm{Dq}=0.6^{*}$ (oxidation state) and a magnetic field of $10 \mathrm{~T}$. The relationship XMCD = right circular polarized - left circular polarized is used as in ref 1 . Prior to this subtraction, all right circular polarized spectra (XAS, 3s-PFY) were normalized to the maximum, and the left circular polarized spectra were divided by the same value, as applied to normalize the right circular polarized spectra.

PFY, one has to carefully interpret experimental data in case the detector is not placed at the correct angle.

\section{THEORETICAL METHODS}

$\mathrm{L}_{2,3}$-edge XAS and 3s-PFY XAS were calculated for several 3d transition-metal ions $\left(\mathrm{Ti}^{3+}, \mathrm{Ti}^{2+}, \mathrm{Cr}^{3+}, \mathrm{Mn}^{3+}, \mathrm{Mn}^{2+}, \mathrm{Fe}^{2+}, \mathrm{Co}^{2+}\right.$, $\left.\mathrm{Ni}^{2+}\right)$ in octahedral $\left(\mathrm{O}_{h}\right)$ symmetry with a fixed crystal-field parameter $10 \mathrm{Dq}=+1.2(+1.8)$ for the divalent (trivalent) ions ${ }^{14}$ with the Quanty code, ${ }^{20-22}$ applying the rule of thumb of $10 \mathrm{Dq}=0.6^{*}$ oxidation state, ${ }^{14}$ which agrees often very well with experimental data with particular formal oxidation states, especially for 3d-metal oxides. ${ }^{1,15}$ The TT-multiplet code developed by Thole ${ }^{23}$ was used to provide the Quanty-input for the Hartree-Fock-Slater integrals $(2 \mathrm{p}-3 \mathrm{~d}, 2 \mathrm{p}-3 \mathrm{~s}, 3 \mathrm{~s}-3 \mathrm{~d}$, and $3 d-3 d$ ) scaled to $80 \%$ and for the atomic $3 \mathrm{~d}$ spin-orbit coupling. For the XMCD simulations with Quanty, a magnetic field contribution with $B=10 \mathrm{~T}$ was added to the ground and X-ray excited-state Hamiltonians.

As an additional check, also $\mathrm{L}_{2,3}$-edge XAS and 3s-PFY for these $3 \mathrm{~d}$ transition-metal ions were calculated with the CTM4XAS 5.2 interface. ${ }^{1,24}$ The CTM4XAS 3s-PFY XAS calculations were performed in two steps: First, the X-ray excitation $2 p$ to $3 d\left(2 p^{6} 3 d^{n} \rightarrow 2 p^{5} 3 d^{n+1}\right)$ was simulated with the
CTM4XAS 5.2 interface, while the $3 \mathrm{~s}$ to $2 \mathrm{p}$ emission was constructed with the TT-multiplet programs ${ }^{12,23}$ based on Cowan's code. $^{25}$ The effect of interference in the RIXS intermediate state on the 3s-PFY simulations was tested for the $\mathrm{Fe}^{2+}$ ion with $10 \mathrm{Dq}=1.2 \mathrm{eV}$. The simulations with and without RIXS interference did not show any spectral differences in the 3s-PFY result. However, the inclusion of interference in RIXS required substantially more computational time. Both constructed files were combined in the CTM4RIXS interface to create RIXS maps, similar to previous procedures. ${ }^{8,11}$ The integrated emission as a function of excitation energy in the CTM4RIXS interface then yields the simulated 3s-PFY-XAS. In this report, only simulations performed with Quanty are shown, but the CTM4XAS simulations lead to the same results.

\section{ASSOCIATED CONTENT}

\section{S Supporting Information}

The Supporting Information is available free of charge on the ACS Publications website at DOI: 10.1021/acs.jpclett.8b00720.

Comparison of $3 \mathrm{~s}-\mathrm{PFY}$ and XAS in the isotropic approximation $(X+Y+Z)$ polarizations in combined and $(X+Y+Z)$ fluorescence out combined for the $3 \mathrm{~d}$ series from $3 \mathrm{~d}^{1}$ to $3 \mathrm{~d}^{8}$ (Figure $\mathrm{S} 1$ ) and a comparison of $3 \mathrm{~s}-\mathrm{PFY}$ and XAS with horizontal and vertical polarized incoming beam and isotropic fluorescence (Figure S2). (PDF)

\section{AUTHOR INFORMATION}

\section{Corresponding Author}

*E-mail: p.s.miedema@gmail.com.

\section{ORCID}

Piter S. Miedema: 0000-0003-1959-3216

Martin Beye: 0000-0002-3924-2993

\section{Notes}

The authors declare no competing financial interest.

\section{ACKNOWLEDGMENTS}

P.S.M. thanks Prof. Maurits Haverkort for discussions on Quanty. M.B. and P.S.M are financially supported by the Helmholtz Association via grant VH-NG-1105.

\section{REFERENCES}

(1) de Groot, F.; Kotani, A. Core Level Spectroscopy of Solids; CRC Press, 2008.

(2) Himpsel, F. J. Photon-in Photon-out Soft X-Ray Spectroscopy for Materials Science. Phys. Status Solidi B 2011, 248 (2), 292-298.

(3) Catalysis: An Integrated Approach, 2nd ed.; van Santen, R. A., van Leeuwen, P. W. N. M., Moulijn, J. A., Averill, B. A., Eds.; Elsevier Science B.V.: Amsterdam, The Netherlands, 2000.

(4) van der Laan, G.; Figueroa, A. I. X-Ray Magnetic Circular Dichroism-a Versatile Tool to Study Magnetism. Coord. Chem. Rev. 2014, 277-278, 95-129.

(5) Wadati, H.; Achkar, A. J.; Hawthorn, D. G.; Regier, T. Z.; Singh, M. P.; Truong, K. D.; Fournier, P.; Chen, G.; Mizokawa, T.; Sawatzky, G. A. Utility of the Inverse Partial Fluorescence for Electronic Structure Studies of Battery Materials. Appl. Phys. Lett. 2012, 100, 193906.

(6) de Groot, F. M. F.; Arrio, M. A.; Sainctavit, P.; Cartier, C.; Chen, C. T. Fluorescence Yield Detection: Why It Does Not Measure the XRay Absorption Cross Section. Solid State Commun. 1994, 92 (12), 991-995.

(7) Regier, T. Z.; Achkar, A. J.; Peak, D.; Tse, J. S.; Hawthorn, D. G. Hawthorn, D. G. Dark Channel Fluorescence Observations Result 
from Concentration Effects rather than Solvent-Solute Charge Transfer. Nat. Chem. 2012, 4 (10), 765-768.

(8) Kurian, R.; Kunnus, K.; Wernet, P.; Butorin, S. M.; Glatzel, P.; de Groot, F. M. F. Intrinsic Deviations in Fluorescence Yield Detected XRay Absorption Spectroscopy: The Case of the Transition Metal L2,3 Edges. J. Phys.: Condens. Matter 2012, 24, 452201.

(9) Wernet, P.; Kunnus, K.; Schreck, S.; Quevedo, W.; Kurian, R.; Techert, S.; de Groot, F. M. F.; Odelius, M.; Föhlisch, A. Dissecting Local Atomic and Intermolecular Interactions of Transition-Metal Ions in Solution with Selective X-Ray Spectroscopy. J. Phys. Chem. Lett. 2012, 3, 3448-3453.

(10) Soldatov, M. A.; Lange, K. M.; Gotz, M. D.; Engel, N.; Golnak, R.; Kothe, A.; Aziz, E. F. On the Origin of Dips in Total Fluorescence Yield X-Ray Absorption Spectra: Partial and Inverse Partial Fluorescence Yield at the L-Edge of Cobalt Aqueous Solution. Chem. Phys. Lett. 2012, 546, 164-167.

(11) Miedema, P. S.; Wernet, P.; Föhlisch, A. State-Dependent Fluorescence Yields through the Core-Valence Coulomb Exchange Parameter. Phys. Rev. A: At., Mol., Opt. Phys. 2014, 89, 52507.

(12) Thole, B. T.; van der Laan, G. Branching Ratio in X-Ray Absorption Spectroscopy. Phys. Rev. B: Condens. Matter Mater. Phys. 1988, 38 (5), 3158-3171.

(13) Golnak, R.; Xiao, J.; Atak, K.; Unger, I.; Seidel, R.; Winter, B.; Aziz, E. F. Undistorted X-Ray Absorption Spectroscopy Using S-CoreOrbital Emissions. J. Phys. Chem. A 2016, 120, 2808-2814.

(14) Miedema, P. S. X-Ray Spectroscopy of Inorganic Materials; Utrecht University, 2012.

(15) Haverkort, M. W.; Hollmann, N.; Krug, I. P.; Tanaka, A. Symmetry Analysis of Magneto-Optical Effects: The Case of X-Ray Diffraction and X-Ray Absorption at the Transition Metal L_ $\{2,3\}$ Edge. Phys. Rev. B: Condens. Matter Mater. Phys. 2010, 82 (9), 94403.

(16) Liu, B.; Piamonteze, C.; Delgado-Jaime, M. U.; Wang, R.-P.; Heidler, J.; Dreiser, J.; Chopdekar, R.; Nolting, F.; de Groot, F. M. F. Sum Rule Distortions in Fluorescence-Yield X-Ray Magnetic Circular Dichroism. Phys. Rev. B: Condens. Matter Mater. Phys. 2017, 96, 54446.

(17) Uhlig, J.; Doriese, W. B.; Fowler, J. W.; Swetz, D. S.; Jaye, C.; Fischer, D. A.; Reintsema, C. D.; Bennett, D. A.; Vale, L. R.; Mandal, U.; et al. High-Resolution X-Ray Emission Spectroscopy with Transition-Edge Sensors: Present Performance and Future Potential. J. Synchrotron Radiat. 2015, 22, 766-775.

(18) Marschall, F.; Yin, Z.; Rehanek, J.; Beye, M.; Döring, F.; Kubiček, K.; Raiser, D.; Veedu, S. T.; Buck, J.; Rothkirch, A.; et al. Transmission Zone Plates as Analyzers for Efficient Parallel 2D RIXSMapping. Sci. Rep. 2017, 7, 8849.

(19) Mitzner, R.; Rehanek, J.; Kern, J.; Gul, S.; Hattne, J.; Taguchi, T.; Alonso-mori, R.; Tran, R.; Weniger, C.; Schröder, H.; et al. L-edge $\mathrm{X}$-ray Absorption Spectroscopy of Dilute Systems Relevant to Metalloproteins Using an X-Ray Free-Electron Laser. J. Phys. Chem. Lett. 2013, 4, 3641-3647.

(20) Haverkort, M. W. Quanty for Core Level Spectroscopy Excitons, Resonances and Band Excitations in Time and Frequency Domain. J. Phys.: Conf. Ser. 2016, 712, 012001.

(21) Haverkort, M. W.; Zwierzycki, M.; Andersen, O. K. Multiplet Ligand-Field Theory Using Wannier Orbitals. Phys. Rev. B: Condens. Matter Mater. Phys. 2012, 85 (16), 165113.

(22) Haverkort, M. W.; Sangiovanni, G.; Hansmann, P.; Toschi, A.; Lu, Y.; Macke, S. Bands, Resonances, Edge Singularities and Excitons in Core Level Spectroscopy Investigated within the Dynamical MeanField Theory. EPL 2014, 108, 57004.

(23) Thole, B. T.; Cowan, R. D.; Sawatzky, G. A.; Fink, J.; Fuggle, J. C. New Probe for the Ground-State Electronic Structure of NarrowBand and Impurity Systems. Phys. Rev. B: Condens. Matter Mater. Phys. 1985, 31 (10), 6856-6858.

(24) Stavitski, E.; de Groot, F. M. F. The CTM4XAS Program for EELS and XAS Spectral Shape Analysis of Transition Metal L Edges. Micron 2010, 41, 687-694.

(25) Cowan, R. D. The Theory of Atomic Structure and Spectra; University of California Press: Berkely, CA, 1981. 